\title{
Ischemic postconditioning promotes left ventricular functional recovery after cardioplegic arrest in an in vivo piglet model of global ischemia reperfusion injury on cardiopulmonary bypass
}

\author{
Gen Shinohara, MD, Kiyozo Morita, MD, PhD, Ryuichi Nagahori, MD, PhD, Yoshihiro Koh, MD, PhD, \\ Katsushi Kinouchi, MD, Takayuki Abe, MD, and Kazuhiro Hashimoto, MD, PhD
}

\begin{abstract}
Objective: An in vivo study of piglets on cardiopulmonary bypass was performed to determine whether postconditioning has a cardioprotective effect after cardioplegic arrest in large animals.

Methods: Eighteen piglets were subjected to 90 minutes of cardioplegic arrest followed by 30 minutes of reperfusion. In 6 animals (control), there was no intervention at reperfusion. In 6 other animals, 6 cycles of unclamping and reclamping for 10 seconds each were done before reperfusion (postconditioning 10), whereas 3 cycles of unclamping and reclamping for 30 seconds each were performed in another 6 piglets (postconditioning 30 ).
\end{abstract}

\begin{abstract}
Results: Recovery of left ventricular contractility and diastolic function (percent of preischemic value) was significantly better in both postconditioning groups (contractility: $89.2 \%$ and 118.2 ; diastolic function: $142.3 \%$ and 120.4; in the postconditioning 10 and 30 groups, respectively) compared with the control (contractility: $46.1 \%$; diastolic function: $218.5 \%$ ). Recovery of global cardiac function (ventricular function curve analysis) was improved only in the postconditioning 30 group. Troponin-T release during reperfusion was significantly reduced in the postconditioning 10 group compared with all groups (plasma troponin- $T$ was $0.58 \mathrm{ng} / \mathrm{mL}$ in postconditioning 10, 1.85 in postconditioning 30 , and 2.54 in control). The myocardial lipid peroxide was significantly higher in the control group than in both postconditioning groups after reperfusion $(199 \%$ vs $112 \%$ and $131 \%$ ).
\end{abstract}

Conclusions: Both postconditioning algorisms promoted functional recovery after cardioplegic arrest in a large animal model along with the limitation of lipid peroxidation with or without the reduction of troponin-T release. (J Thorac Cardiovasc Surg 2011;142:926-32)

In 2003, ischemic postconditioning was first introduced by Zhao and associates ${ }^{1}$ as a potent method of endogenous cardioprotection against ischemia-reperfusion injury. Postconditioning by several brief cycles of coronary artery reperfusion and reocclusion, when performed immediately at the onset of reperfusion, has a universally protective effect against myocardial infarction in coronary ligation models. ${ }^{1-4}$ However, the influence of postconditioning on another major consequence of ischemia-reperfusion injury, that is, myocardial dysfunction, is still controversial. In an in vivo coronary ligation model, postconditioning did not show any protective effect against deterioration of regional and global cardiac function parameters, such as left ventricular (LV) end-diastolic pressure (LVEDP), the maximum positive and negative derivatives of LV pressure $( \pm \mathrm{dP} / \mathrm{dt}$ max $)$, systolic shortening, and segmental work, despite an infarct-sparing

From the Department of Cardiac Surgery, Jikei University School of Medicine, Tokyo, Japan.

Disclosures: Authors have nothing to disclose with regard to commercial support.

Received for publication June 16, 2010; revisions received Sept 29, 2010; accepted for publication Jan 19, 2011; available ahead of print Feb 28, 2011.

Address for reprints: Kiyozo Morita, MD, PhD, 3-25-8 Nishishinbashi, Minato-ku,

Tokyo, 105-8461, Japan (E-mail: kiyozo@jikei.ac.jp).

$0022-5223 / \$ 36.00$

Copyright (c) 2011 by The American Association for Thoracic Surgery

doi:10.1016/j.jtcvs.2011.01.028 effect being consistently observed in various species (dogs, ${ }^{1}$ rats, ${ }^{2}$ and rabbits $\left.{ }^{5}\right)$. Another study that used a short ischemic period (10 minutes) failed to demonstrate any antistunning effect of postconditioning on depressed regional wall motion (LV wall thickening in dogs or segmental shortening in rabbits). ${ }^{3}$ More recently, a beneficial effect of postconditioning on LV function after 30 minutes of global ischemia (+dP/dt max, LVEDP, and recovery of developed LV pressure) was demonstrated in an isolated rat heart preparation., ${ }^{4,6}$

However, the value of postconditioning in large animals subjected to cardioplegic arrest of a longer duration while supported by cardiopulmonary bypass (CPB) has not been investigated.

Therefore, we conducted a study of postconditioning in the surgical setting that focused on protection against postischemic myocardial dysfunction. Our purpose was to assess the cardioprotective effect of postconditioning after prolonged cardioplegic arrest in piglets on CPB and to determine the impact of different postconditioning algorithms on LV functional recovery.

\footnotetext{
MATERIALS AND METHODS

All experimental animals received humane care in compliance with the "Guide for the Care and Use of Laboratory Animals" (National Institutes of Health Publication No. 85-23, revised 1996).
} 


\section{Abbreviations and Acronyms \\ $\mathrm{CPB}=$ cardiopulmonary bypass \\ $\pm \mathrm{dP} / \mathrm{dt}=$ maximum positive and negative \\ $\max$ derivatives of left ventricular pressure \\ Ees = end-systolic elastance \\ LAP $=$ left atrial pressure \\ $\mathrm{LV}=$ left ventricular \\ LVEDP $=$ left ventricular end-diastolic pressure \\ LVSWI = left ventricular stroke work index \\ MAP = mean arterial pressure \\ Tau $=$ time constant of ventricular relaxation}

\section{Experimental Preparation}

Eighteen Large White-Landrace-Duroc piglets (weighing 9-12 kg, 4-6 weeks old) were initially anesthetized with intravenous ketamine hydrochloride $(4 \mathrm{mg} / \mathrm{kg})$. After endotracheal intubation, each piglet was ventilated with a volume-cycled respirator (PH-5F II; Acoma Medical Industry Co, Ltd, Tokyo, Japan) using oxygen, air, and $1 \%$ to $1.5 \%$ sevoflurane, while maintaining carbon dioxide and $\mathrm{pH}$ within the normal range. The electrocardiogram was recorded with surface electrodes.

A catheter was inserted into the left femoral vein to monitor central venous pressure, to infuse acetated Ringer solution and anesthetic intravenously, and to withdraw blood samples. Both $0.3 \mathrm{mg} / \mathrm{kg}$ of pancuronium bromide and $0.3 \mathrm{~mL} / \mathrm{kg}$ of heparin sodium were infused. Another catheter was placed in the left femoral artery to monitor arterial pressure.

After midline sternotomy, the pericardium was opened. LV dimensions were measured in 2 axes (anterior-posterior and apex-base) by pairs of ultrasonic transducers that were positioned on the epicardial surface and secured with purse-string sutures. A Millar Mikro-Tip catheter (Millar Instruments, Inc, Houston, Tex) was inserted into the left ventricle through the apex for measurement of the LV pressure. The ultrasonic crystals were attached to a digital sonomicrometer (Sonometrics Corporation, London, Ontario, Canada). LV pressure and data from the crystals were monitored continuously on line by computer and were intermittently recorded for off-line analysis.

A $12 \mathrm{~F}$ arterial cannula was placed in the aortic arch via the right carotid artery and a $24 \mathrm{~F}$ cannula was placed in the right atrium. Then a snare was passed around the inferior vena cava. An $8 \mathrm{~F}$ cannula was advanced into the left atrium through left atrial appendage for monitoring of left atrial pressure (LAP) and for venting the left side of the heart during cardiac arrest. Another $8 \mathrm{~F}$ catheter was placed in the main pulmonary artery for additional venting. An aortic root cannula with side branches for administration of cardioplegic solution, pressure monitoring, and venting was inserted into the aortic root.

The CPB circuit was primed with heparinized homologous whole blood drawn from a donor pig, acetated Ringer solution, hydroxyethylated starch, D-mannitol, and sodium bicarbonate, followed by hemoconcentration with extracorporeal ultrafiltration to achieve an intrabypass hematocrit of $20 \%$ to $25 \%$. All hemodynamic parameters were monitored simultaneously on a 6-channel polygraph system. A transit-time ultrasonic flow meter (T201; Transonic System, Inc, Ithaca, NY) was intermittently placed around the ascending aorta for measurement of cardiac output and data were recorded simultaneously with central venous pressure, LAP, and arterial pressure to calculate LV stroke work and to create LV function curves for analysis.

\section{Experimental Protocol}

After heparinization $(0.3 \mathrm{~mL} / \mathrm{kg}), \mathrm{CPB}$ was initiated at a flow rate of approximately $2.2 \mathrm{~L} \cdot \min ^{-1} \cdot \mathrm{m}^{-2}$. We used a pulsatile roller pump
(Advanced Perfusion System 1; Terumo Corp, Tokyo, Japan) and a membrane oxygenator (Capiox SX10; Terumo Corp). Arterial blood pressure was maintained at 50 to $80 \mathrm{~mm} \mathrm{Hg}$, with the $\mathrm{pH}$ at 7.35 to 7.45 , oxygen tension greater than $300 \mathrm{~mm} \mathrm{Hg}$, carbon dioxide tension at 35 to $45 \mathrm{~mm}$ $\mathrm{Hg}$, and systemic normothermia.

The aortic crossclamp was applied and cardioplegic arrest was initiated with $400 \mathrm{~mL}$ of St Thomas Hospital II solution (Miotector; Mochida Corp, Japan) being infused within 3 minutes at an aortic root pressure of 50 to 80 $\mathrm{mm} \mathrm{Hg}$.

Then the heart was subjected to 90 minutes of global ischemia with topical pericardial cooling (ice slush) and without any additional cardioplegic solution.

No inotropic or vasoactive drugs were used. The heart was kept in the empty beating state for 30 minutes after reperfusion, and then the piglet was weaned from CPB. After postischemic cardiac function measurements, blood sampling, and final tissue sampling, the animal died by exsanguination after removal of the heart under anesthesia.

\section{Experimental Groups}

The piglets were divided into the following groups ( 3 male and 3 female were in each group) on the basis of the method of reperfusion (Figure 1). Uncontrolled reperfusion group (control). In 6 piglets, the crossclamp was simply removed, and the heart was reperfused at a perfusion pressure of 50 to $70 \mathrm{~mm} \mathrm{Hg}$.

Postconditioning groups. Postconditioning 10 group. In 6 piglets, 6 cycles of unclamping and reclamping of the aorta for 10 seconds each were performed over 2 minutes before complete removal of the crossclamp.

Postconditioning 30 group. In 6 piglets, 3 cycles of unclamping and reclamping of the aorta for 30 seconds each over 3 minutes were performed before complete removal of the crossclamp.

\section{Myocardial Performance}

LV pressure-volume loops were drawn from pressure data measured by a Millar catheter-tip manometer and sonomicrometry data using a 2-axis ellipsoidal model, while cardiac output was measured with a flow meter at baseline and after 30 minutes of reperfusion. A series of pressure-volume loops was generated through transient occlusion of the inferior vena cava by tightening and releasing the tape snare during an 8 -second period of apnea. Post hoc analysis of cardiac function was done with the Sonometrics software package.

LV contractility. LV contractility was assessed from the descending slope of the end-systolic pressure-volume relationship by linear regression analysis and was designated as end-systolic elastance (Ees).

Diastolic function. The time constant of ventricular relaxation (Tau) was calculated from the decline of the LV pressure trace at a stable LVEDV during isovolumic relaxation.

Ees and Tau values measured after reperfusion were expressed as a percentage of the respective baseline values.

\section{Global Cardiac Performance From the LV Function Curve}

Integrated myocardial performance was evaluated by calculating the LV stroke work index (LVSWI) after infusing blood through the arterial cannula and simultaneously recording the mean arterial pressure (MAP), LAP, and heart rate (HR), as well as measuring cardiac output (CO) (flow meter). LVSWI was calculated with the following equation:

$$
\begin{aligned}
\operatorname{LVSWI}\left(\mathrm{g} \cdot \mathrm{m}^{-1} \cdot \mathrm{kg}^{-1}\right)= & (\mathrm{MAP}-\mathrm{LAP}) \times \mathrm{CO}(\mathrm{mL} / \mathrm{min}) \\
& \times 0.0135 /(\mathrm{HR} \times \mathrm{BW}[\mathrm{kg}])
\end{aligned}
$$

where BW is body weight. 


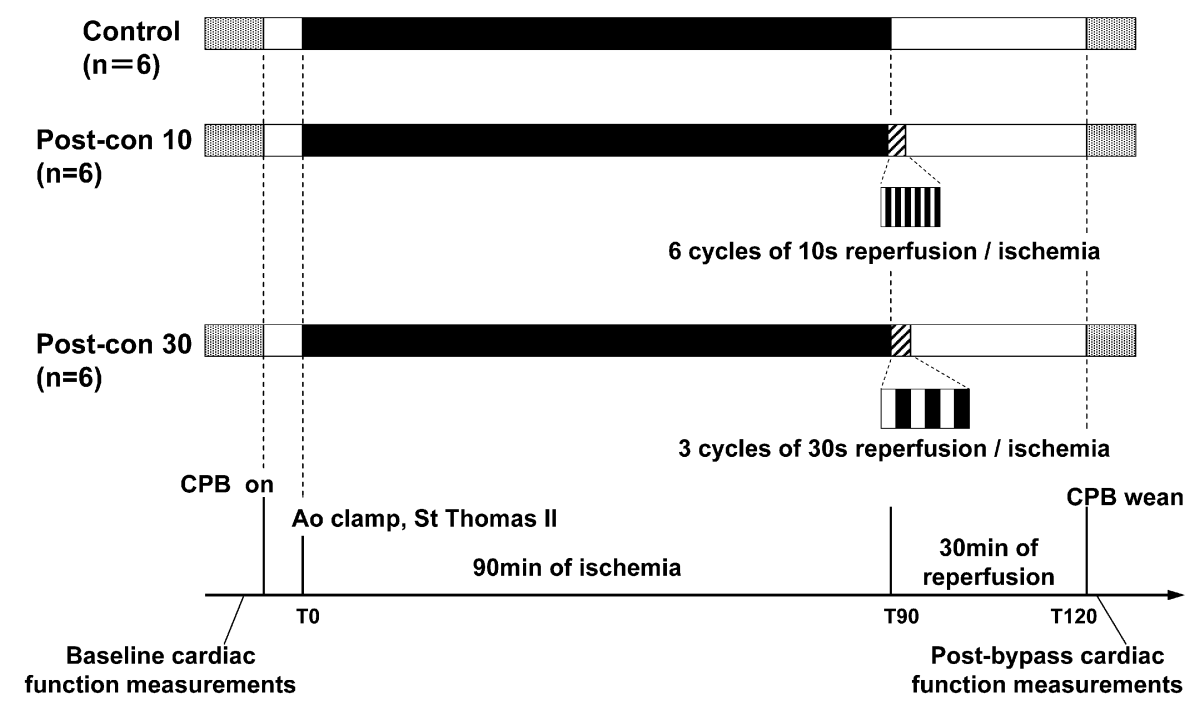

FIGURE 1. Experimental protocols of the different groups. All piglets were subjected to 90 minutes of cardioplegic arrest under cardiopulmonary bypass $(C P B)$. In the control group, there was no intervention before 30 minutes of reperfusion. The postconditioning (Post-con) groups received 6 cycles of 10 seconds $(s)$ of reperfusion/10 seconds of ischemia (Post-con 10) or 3 cycles of 30 seconds of reperfusion/30 seconds of ischemia (Post-con 30) at the start of 30 minutes of reperfusion. Cardiac function was measured before CPB and at 30 minutes after reperfusion just after weaning from CPB.

Ventricular function curves were drawn by plotting LAP (x axis) versus LVSWI (y axis). Then linear regression analysis was performed and the area of the trapezoid between the regression lines for minimum and maximum LAPs obtained before and after CPB was calculated. Recovery of LV performance was assessed by calculating the area of the trapezoid after reperfusion as a percentage of the baseline value (Figure 2).

\section{Biochemical Parameters}

Plasma troponin-T. Arterial blood samples were taken before initiation of $\mathrm{CPB}$, at the end of ischemia, and after 15 and 30 minutes of reperfusion. Biochemical myocardial injury was determined by measuring the plasma troponin-T level using the electrochemiluminescent immunoassay method.
Tissue lipid peroxide. Transmural samples of the right ventricular free wall were taken before initiation of CPB, at the end of ischemia, and after 30 minutes of reperfusion. These samples were frozen and stored at $-80^{\circ} \mathrm{C}$ until assay.

Then the lipid peroxide content of tissue homogenates was measured by the methylene blue-hemoglobin method.

\section{Statistics}

Continuous variables are expressed as the mean \pm standard deviation.

One-factor factorial analysis of variance or 1-factor repeated-measures analysis of variance was performed to assess the statistical significance of differences in discrete or continuous variables among the groups, respectively. If a significant $\mathrm{F}$ ratio was obtained, further analysis was carried
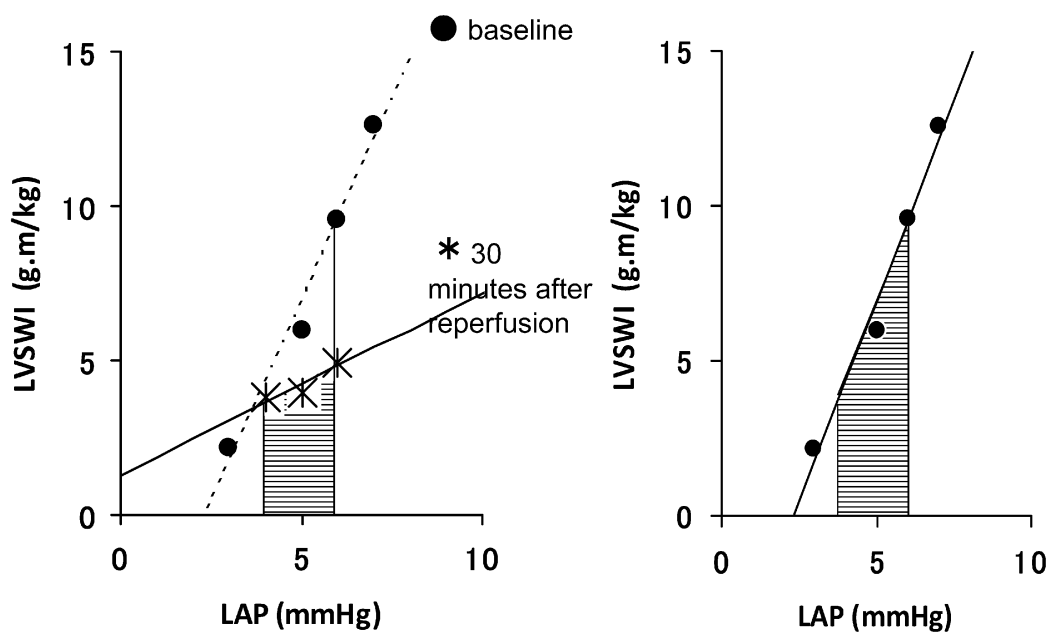

FIGURE 2. Ventricular function curves were created by plotting left atrial pressure (LAP) ( $x$ axis) versus left ventricular stroke work index (LVSWI) (y axis), and linear regression lines were drawn. The area of the trapezoid between the regression lines for the minimum and maximum LAP obtained before and after cardiopulmonary bypass was defined as the area under the left ventricular function curve. 
out with the Scheffé $\mathrm{F}$ post hoc test to identify significant differences between the groups.

\section{RESULTS}

\section{Myocardial Performance}

The results are presented in Figure 3.

Ees. In the control group, there was a marked decrease of Ees after 30 minutes of reperfusion to $46.1 \% \pm 7.1 \%$ of the baseline value, indicating LV contractile dysfunction. In contrast, there was significantly better recovery of Ees in both the postconditioning groups $(89.2 \% \pm 33.5 \%$ in the postconditioning 10 group $[P<.05]$ and $118.2 \% \pm$ $20.3 \%$ in the postconditioning 30 group $[P<.01]$, respectively).

Tau. The control group showed severe increase of Tau after 30 minutes of reperfusion among all of the groups $(218.5 \%$ $\pm 49.7 \%$ of baseline value), indicating diastolic dysfunction. In the postconditioning groups, the increase of Tau was significantly smaller $(142.3 \% \pm 50.4 \%$ in the postconditioning 10 group $[P<.05]$ and $120.4 \% \pm 25.3 \%$ in the postconditioning 30 group $[P<.01])$.

\section{Global LV Performance}

In the control group, the LV function curve area after 30 minutes of reperfusion was $28.6 \% \pm 4.0 \%$ of baseline, indicating severe hemodynamic impairment attributable to LV systolic and diastolic dysfunction. In the postconditioning 30 group, there was significant improvement of the curve area compared with the control group $(69.4 \% \pm$ $28.4 \% ; P<.05)$, indicating that the protective effect on LV performance resulted from both systolic and diastolic functional recovery. In the postconditioning 10 group, a tendency to improvement of LVSWI was shown with marginal significance $(P=.084)$.

\section{Biochemical Parameters}

The profile of plasma troponin-T levels over time is presented in Figure 4. There was a marked increase of troponin-T after reperfusion in the control group $(2.54 \pm$ $0.81 \mathrm{ng} / \mathrm{mL}$ ), whereas troponin-T was significantly lower after 30 minutes of reperfusion in the postconditioning 10 group $(0.58 \pm 0.25 \mathrm{ng} / \mathrm{mL} ; P<.05$ vs all groups $)$. In the postconditioning 30 group, however, a reduction of troponin- $\mathrm{T}$ was not seen, despite better functional recovery in this group $(1.85 \pm 0.4 \mathrm{ng} / \mathrm{mL})$. Right ventricular myocardial lipid peroxide levels are presented in Table 1.

There were no significant differences of lipid peroxide levels before CPB and after 90 minutes of ischemia among the 3 groups. There was a dramatic increase of lipid peroxide
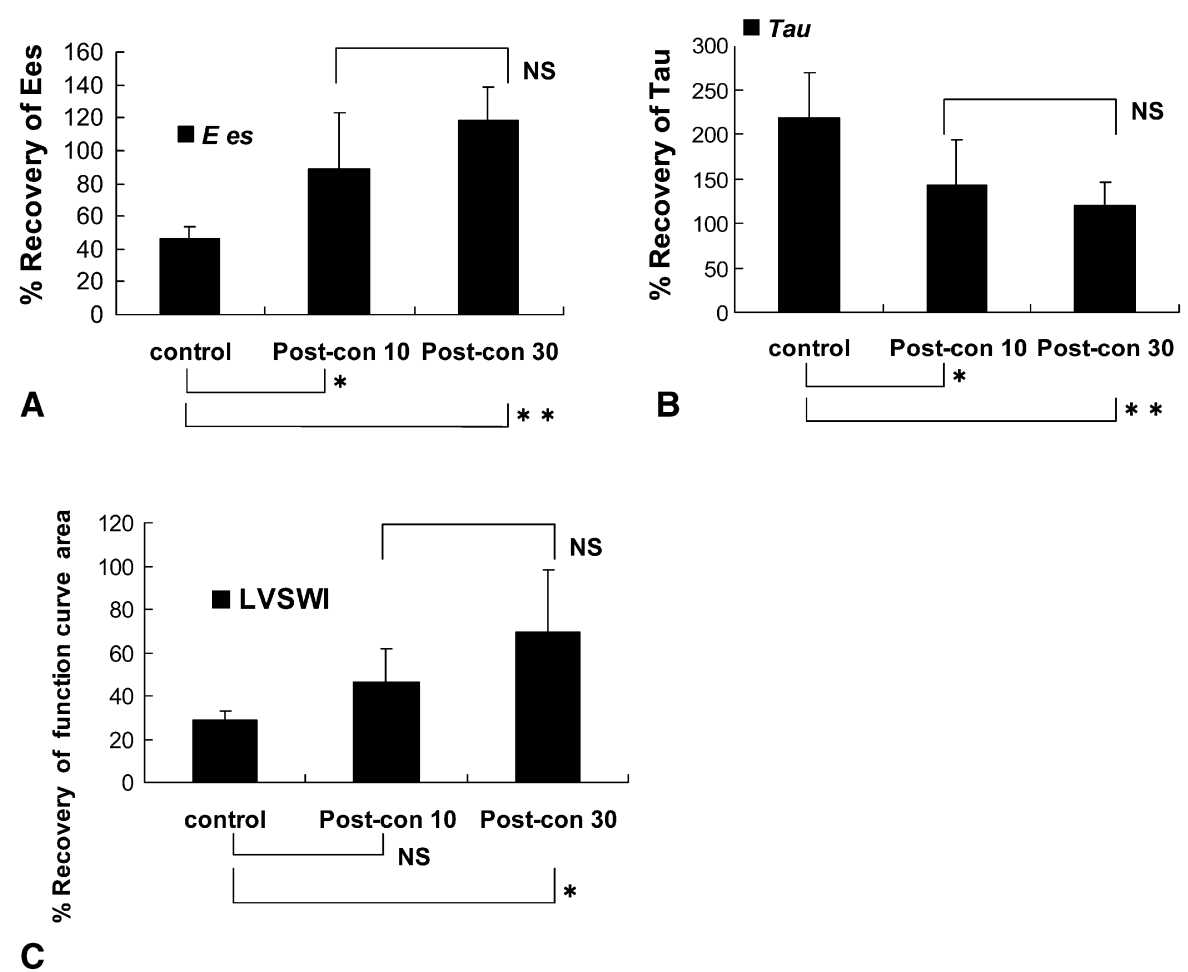

FIGURE 3. (A) End-systolic elastance (Ees), (B) time constant of isovolumic relaxation (Tau), (C) and left ventricular stroke work index ( $L V S W I$ ) after 30 minutes of reperfusion. Each parameter is shown as the percent recovery from the preischemic value. Data are reported as the mean \pm standard error of the mean. $* P<.05, * * P<.01$ versus control group. There are no significant differences in Ees, Tau, and LVSWI between 2 postconditioning groups. There is not statically significant, but marginally significant, improvement of LVSWI in the postconditioning (Post-con) 10 group compared with control $(P=.084)$. NS, Not significant. 


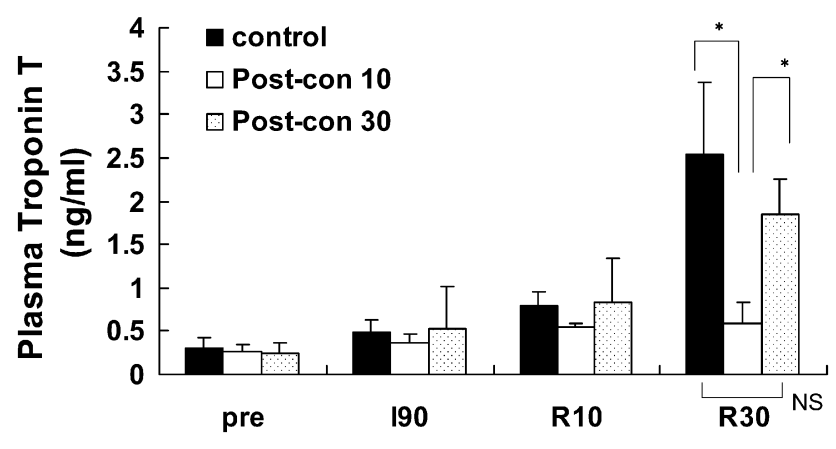

FIGURE 4. Changes of the plasma troponin-T levels. Post-con, Postconditioning; $N S$, not significant; $R$, reperfusion. $* P<.05$ versus all groups.

after reperfusion in the control group $(199 \% \pm 56 \%$ of the value after 90 minutes of ischemia). The increase of lipid peroxide after 30 minutes of reperfusion (as a percentage of the value at the end of ischemia) was significantly smaller in both the postconditioning groups compared with the control group $(112 \% \pm 39 \%$ for postconditioning 10 and $131 \% \pm 10 \%$ for postconditioning 30 ).

\section{DISCUSSION}

In the present study, we demonstrated that performance of ischemic postconditioning before complete aortic unclamping led to improved postbypass LV performance in piglets after cardioplegic arrest. Postconditioning ameliorated LV contractile dysfunction and isovolumic diastolic dysfunction, leading to restoration of nearly normal global $\mathrm{LV}$ performance, along with the reduction of myocardial lipid peroxide levels (an indicator of cellular oxidative injury).

Previous studies in coronary artery occlusion-reperfusion models have shown that postconditioning ameliorates multiple manifestations of ischemia-reperfusion injury, including a reduction of infarct size and endothelial dysfunction. ${ }^{1,2,7}$

Nevertheless, the effect of postconditioning on myocardial dysfunction remains controversial. Studies of in vivo coronary ligation models in various species have revealed a universal protective effect against myocardial infarction

TABLE 1. Myocardial lipid peroxide level after 30 minutes of reperfusion.

\begin{tabular}{lccc}
\hline & Control & Post-con 10 & Post-con 30 \\
\hline Pre $(\mathrm{ng} / \mathrm{mL})$ & $0.0183 \pm 0.0082$ & $0.0296 \pm 0.0267$ & $0.0140 \pm 0.0015$ \\
Ischemia & $0.0195 \pm 0.0096$ & $0.0269 \pm 0.0187$ & $0.0144 \pm 0.0012$ \\
$\quad 90(\mathrm{ng} / \mathrm{mL})$ & & & \\
Reperfusion & $0.0382 \pm 0.0213$ & $0.0247 \pm 0.0038$ & $0.0191 \pm 0.0026$ \\
$\quad 30(\mathrm{ng} / \mathrm{mL})$ & & & \\
$\mathrm{R} 30 / \mathrm{I} 90(\%)$ & $199.75 \pm 56.3^{*}$ & $112.18 \pm 39.1^{*}, \dagger$ & $131.96 \pm 10.0^{*}$ \\
\hline
\end{tabular}

$R 30 / 190$, Value at 30 minutes after reperfusion expressed as percentage of the 90-minute ischemia value (measured just before reperfusion). $* P<.05$ versus control group. $\nmid$ There is no significant difference between the 2 postconditioning groups. but have found no protective effect against depression of hemodynamic parameters (MAP, rate pressure product, and LVEDP), global myocardial dysfunction (LVEDP, $+\mathrm{dP} / \mathrm{dt},-\mathrm{dP} / \mathrm{dt}$ ), or impaired regional wall motion (systolic shortening and segmental work) in dogs, ${ }^{1}$ rats, ${ }^{2}$ and rabbits. ${ }^{5}$ Furthermore, experiments with a short ischemic period of 10 minutes for stunning have shown that ischemic postconditioning does not change the depression of LV wall thickening (in dogs) or segmental shortening (in rabbits) over 3 to 24 hours after reperfusion. ${ }^{3}$ In contrast to these studies on models of regional ischemia, recent experimental studies of isolated rat hearts subjected to global ischemia demonstrated that postconditioning can improve $\mathrm{LV}$ dysfunction based on $\mathrm{LV}$ pressure data $(+\mathrm{dP} / \mathrm{dt}$, LVEDP, and recovery of developed LV pressure)., However, unlike the protective effect on ventricular contracture that is well correlated with the reduction of infarct size, the beneficial effect on postischemic systolic dysfunction was reported to show no clear relation to the effect on infarction.

Previous clinical investigations of postconditioning in patients undergoing cardiac surgery in China did not obtain any data on cardiac function. ${ }^{8,9}$

The main mediators and effectors involved in the mechanism of postconditioning are reported to be adenosine, ${ }^{10-14}$ nitric oxide, ${ }^{15}$ a reperfusion injury salvage kinase pathway (phosphatidylinosito 13-kinase and extracellular signalregulated kinase), ${ }^{15,16}$ opening of mitochondrial potassium adenosine triphosphate channels, ${ }^{5}$ and prevention of the mitochondrial permeability transition, ${ }^{17}$ which is a crucial event in reperfusion injury triggered by $\mathrm{Ca}^{2+}$ overload and excessive production of reactive oxygen species. ${ }^{18,19}$ Cyclosporine A, a specific mitochondrial permeability transition inhibitor, has been demonstrated by Oka and associates ${ }^{20}$ to prevent mitochondrial dysfunction and improve myocardial performance (including both systolic and diastolic function) after cardioplegic arrest in isolated rat hearts.

In the present study, both postconditioning algorithms similarly reduced the myocardial lipid peroxide content after 30 minutes of reperfusion, indicating that one of the effectors of ischemia-reperfusion injury was attenuated, although the actual protective effect was dissimilar.

Previous studies on the protective effect of postconditioning against myocardial infarction in other animal models suggested that (1) the infarct-sparing effect differs among species $^{2,5,7}$ and also between genders ${ }^{4}$ and (2) the postconditioning, particularly in length of ischemia and reperfusion, rather than number of cycles, is species dependent. Several extended algorithms with 3 cycles of 30-second reperfusion and 30-second reocclusion have been applied in dog and rabbit models, ${ }^{5,7}$ whereas 4 cycles of reperfusion and reocclusion for 1 minute each were tested in dogs and rabbits by Couvreur and colleagues. ${ }^{3}$ 
In the present study, 2 postconditioning protocols examined showed disparate protective effects on biochemical injury and myocardial dysfunction. In the postconditioning 10 group, limitation of biochemical injury was associated with recovery of myocardial systolic and diastolic function. On the other hand, in the postconditioning 30 group, myocardial functional recovery was prominent with $70 \%$ of baseline global function being restored despite lack of reduction in troponin-T release. In the isolated heart model, myocardial contracture may be mainly due to myocardial infarction, while recovery of contractile function may depend on alleviation of both myocardial necrosis and stunning. ${ }^{4}$ In addition, functional recovery after cardioplegic arrest is thought to be influenced by myocardial metabolism, which had to recover from an ischemic period characterized by minimum oxygen consumption in our model, unlike global ischemia without cardioplegia or normothermic regional ischemia in a coronary ligation model.

The different functional and biochemical outcomes obtained with our 2 postconditioning algorithms indicate that the pattern of repeated ischemic insults may determine the influence on the cellular energy status, resulting in reversible metabolic depression or moderate injury and irreversible change. After the derangement of metabolism caused by cardioplegic arrest, repeated brief ischemic insults (postconditioning 10) were protective against irreversible myocardial injury but failed to promote prompt functional recovery.

On the other hand, more intensive ischemic stimulation (postconditioning 30) also attenuated myocardial lipid peroxide and improved cellular metabolic recovery compared with the control group, although enzyme release was aggravated.

The excessively great recovery of Ees exceeding baseline value up to $118.2 \% \pm 20.3 \%$ seen in the postconditioning 30 group is difficult to understand. One possibility is speculated that either energy metabolic efficacy including inotropic response or activation-contraction coupling or other would be supranormally stimulated in viable myocardium, based on the independence of Ees from cardiac preload and afterload, evident myocardial cell injury from troponin $\mathrm{T}$ release, and association with improvement of Tau. Whether it is a phenomenon similar to hypercontraction, as seen in nonischemic areas in myocardial infarction, is unknown because of lack of wall motion analysis. As another possible explanation, by the remote postconditioning effect, ${ }^{21,22}$ interaction of neurohormonal factor such as norepinephrine overflow between the heart and a remote organ may be involved in our in vivo model.

\section{Limitations}

In this study, tissue lipid peroxide was measured in the right ventricular myocardium to allow repeated sampling (3 times per experiment). Inasmuch as there was a decrease of lipid peroxide in absolute terms after reperfusion, measurement of LV tissue after reperfusion would also be applicable for comparing the groups.

An ischemia-reperfusion protocol with 90 minutes of ischemia and a single dose of cardioplegic solution, which has been demonstrated to induce severe impairment of myocardial performance (cardiac function curve area of $28 \%$ in the control group) was examined in this study. In the surgical setting, repeated administration of crystalloid or blood cardioplegic solution will minimize ischemia-reperfusion injury in most cases. However, the protective effect of postconditioning observed in this study could be valuable for emergency "rescue" therapy in the situation of unexpectedly prolonged cardioplegic arrest or inadequate cardioprotection.

Inasmuch as multidose blood cardioplegia is widely used because of its superior preservation of myocardial aerobic metabolism and high-energy phosphate stores in cardiac surgery, whether postconditioning would be beneficial for attenuation of ischemia-reperfusion injury after multidose blood cardioplegia remains to be determined.

Although postconditioning is a fairly simple and reproducible procedure as regards implementation of principles of several brief cycles of reperfusion and ischemia, repetitive manipulation of the aortic crossclamp performed in our study increases the risk of aortic dissection and crucial embolism in clinical cardiac surgery, particularly for the patient with a fragile or calcified aorta or mobile plaque in the aorta. Therefore, the technical modification to improve the safety of the surgical postconditioning procedure, such as alternating between normal blood infusion and intermission by the use of blood cardioplegic delivery system before aortic unclamping, would be necessary.

\section{CONCLUSIONS}

Postconditioning was demonstrated to have a protective effect against myocardial dysfunction after cardioplegic arrest in piglets with or without the limitation of biochemical injury according to algorism. However, investigation of postconditioning in a multidose blood cardioplegic arrest model and improvement of the procedure are needed before clinical application.

\section{References}

1. Zhao ZQ, Corvera JS, Halkos ME, Kerendi F, Wang NP, Guyton RA, et al. Inhibition of myocardial injury by ischemic postconditioning during reperfusion: comparison with ischemic preconditioning. Am J Physiol Heart Circ Physiol. 2003;285:H579-88.

2. Kin H, Zhao ZQ, Sun HY, Wang NP, Corvera JS, Halkos ME, et al. Postconditioning attenuates myocardial ischemia-reperfusion injury by inhibiting events in the early minutes of reperfusion. Cardiovasc Res. 2004;62:74-85.

3. Couvreur N, Lucats L, Tissier R, Bize A, Berdeaux A, Ghaleh B. Differential ef fects of postconditioning on myocardial stunning and infarction: a study in conscious dogs and anesthetized rabbits. Am J Physiol Heart Circ Physiol. 2006;291: H1345-50.

4. Penna C, Tullio F, Merlino A, Moro F, Raimondo S, Rastaldo R, et al. Postconditioning cardioprotection against infarct size and post-ischemic 
systolic dysfunction is influenced by gender. Basic Res Cardiol. 2009;104: 390-402.

5. Yang XM, Proctor JB, Cui L, Krieg T, Downey JM, Cohen MV. Multiple, brief coronary occlusions during early reperfusion protect rabbit hearts by targeting cell signaling pathways. J Am Coll Cardiol. 2004;44:1103-10.

6. Pinheiro BB, Fiorelli AI, Gomes OM. Effects of ischemic postconditioning on left ventricular function of isolated rat hearts. Rev Bras Cir Cardiovasc. 2009; 24:31-7.

7. Zhao ZQ, Corvera JS, Wang NP, Guyton RA, Vinten-Johansen J. Reduction of infarct size and preservation of endothelial function by ischemic postconditioning: comparison with ischemic preconditioning. Circulation. 2002;106(Suppl):II314.

8. Luo W, Li B, Chen R, Huang R, Lin G. Effect of ischemic postconditioning in adult valve replacement. Eur J Cardiothorac Surg. 2008;33:203-8. Epub 2007 Dec 19.

9. Li B, Chen R, Huang R, Luo W. Clinical benefit of cardiac ischemic postconditioning in corrections of tetralogy of Fallot. Interact Cardiovasc Thorac Surg. 2009;8:17-21. Epub 2008 Oct 14.

10. Xu Z, Downey JM, Cohen MV. Amp 579 reduces contracture and limits infarction in rabbit heart by activating adenosine A2 receptors. J Cardiovasc Pharmacol. 2001;38:474-81.

11. Smits GJ, McVey M, Cox BF, Perrone MH, Clark KL. Cardioprotective effects of the novel adenosine A1/A2 receptor agonist AMP 579 in a porcine model of myocardial infarction. J Pharmacol Exp Ther. 1998;286:611-8.

12. Budde JM, Velez DA, Zhao Z, Clark KL, Morris CD, Muraki S, et al. Comparative study of AMP579 and adenosine in inhibition of neutrophil-mediated vascular and myocardial injury during $24 \mathrm{~h}$ of reperfusion. Cardiovasc Res. 2000;47:294-305.

13. Kis A, Baxter GF, Yellon DM. Limitation of myocardial reperfusion injury by AMP579, an adenosine A1/A2A receptor agonist: role of $\mathrm{A} 2 \mathrm{~A}$ receptor and Erk1/2. Cardiovasc Drugs Ther. 2003;17:415-25.
14. Xu Z, Yang X-M, Cohen MV, Neumann T, Heusch G, Downey JM. Limitation of infarct size in rabbit hearts by the novel adenosine receptor agonist AMP579 administered at reperfusion. J Mol Cell Cardiol. 2000;32: 2339-47.

15. Yang X-M, Kreig T, Cui L, Downey JM, Cohen MV. NECA and bradykinin at reperfusion reduce infarction in rabbit hearts by signaling through PI3K, ERK, and NO. J Mol Cell Cardiol. 2004;36:411-21.

16. Tsang A, Hausenloy DJ, Mocanu MM, Yellon DM. Postconditioning: a form of "modified reperfusion" protects the myocardium by activating the phosphatidylinositol 3-kinase-akt pathway. Circ Res. 2004;95:230-2.

17. Argaud L, Gateau-Roesch O, Raisky O, Loufouat J, Robert D, Ovize M. Postconditioning inhibits mitochondrial permeability transition. Circulation. 2005;111: 194-7. Epub 2005 Jan 10.

18. Piper HM, Meuter K, Schafer C. Cellular mechanism of ischemia-reperfusion injury. Ann Thorac Surg. 2003;75:S644-8.

19. Di Lisa F, Menabò R, Canton M, Barile M, Bernardi P. Opening of the mitochondrial permeability transition pore causes depletion of mitochondrial and cytosolic $\mathrm{NAD}+$ and is a causative event in the death of myocytes in postischemic reperfusion of the heart. J Biol Chem. 2001;276:2571-5.

20. Oka N, Wang L, Mi W, Caldarone CA. Inhibition of mitochondrial remodeling by cyclosporine A preserves myocardial performance in a neonatal rabbit model of cardioplegic arrest. J Thorac Cardiovasc Surg. 2008;135:585-93.

21. Kerendi F, Kin H, Halkos ME, Jiang R, Zatta AJ, Zhao ZQ, et al. Remote postconditioning. Brief renal ischemia and reperfusion applied before coronary artery reperfusion reduces myocardial infarct size via endogenous activation of adenosine receptors. Basic Res Cardiol. 2005;100:404-12.

22. Andreka G, Vertesaljai M, Szantho G, Font G, Piroth Z, Fontos G, et al. Remote ischaemic postconditioning protects the heart during acute myocardial infarction in pigs. Heart. 2007;93:749-52. 